\title{
Influence of the Temperature on the Efficiency of Cellulose Treatment Using Copolymer Chitosan-Eugenol
}

\author{
Olivera Sauperl, Jasna Tompa, Julija Volmajer-Valh \\ Faculty of Mechanical Engineering, Maribor, Slovenia SLOVENIA \\ Correspondence to: \\ Olivera Sauperl email: olivera.sauperl@um.si
}

\begin{abstract}
In order to achieve effective antimicrobial protection of textile materials against microorganisms, a natural compound called chitosan has become very interesting. In regard to the antimicrobial protection of textile materials, functionalization with chitosan does not affect some other properties, such as antioxidative or any other action. For this reason, it seems appropriate for chitosan to be combined with any natural antimicrobial active compound, such as eugenol, an extract of clove oil. During this research viscose as a representative of cellulose fibers was used, because it can be functionalized relatively easily. In terms of functionalization, the drying temperature of viscose, after antimicrobial compound application onto substrate, as well as the successful synthesis of copolymer chitosan/eugenol is also important. FTIR spectroscopy was used to evaluate the efficiency of synthesizing a chitosan/eugenol graft copolymer. The spectrophotometric method Acid Orange 7 was chosen as a means for determining the proportion of available antimicrobial active amino groups. In addition, microbiological testing of selected pathogenic micro-organisms was also performed. The results were compared with the results for viscose functionalized by a $1 \%$ solution of chitosan.
\end{abstract}

Keywords: chitosan, eugenol, functionalization, viscose, FTIR spectroscopy, Acid orange 7, antimicrobial

\section{INTRODUCTION}

The presented work deals with viscose functionalization by using copolymer chitosaneugenol in order to protect viscose against microorganisms [1-3]. Micro-organisms present on textiles are primarily bacteria and algae which, in a case where the textile material is located within a very humid environment, excessively multiply. Therefore, this represents a food-source for various microorganisms which, in this way, have even more favorable conditions for further reproduction. The presence of fungi is also an inconvenience as they are known to cause damage to the fibers, as well as stains on the material as a result of the material's decomposition. Further adverse impact from the bacteria is reflected in the textiles' unpleasant smell and mucus touch, which is unacceptable as usable properties of the textile materials [4].

Therefore, within textile materials there is a symbiotic relation between algae, fungi, and bacteria, so it is desirable to also treat textile materials against these various micro-organisms. Antimicrobial protection is inevitable, especially in the case of protective clothing exposed to infections from pathogenic microorganisms in hospitals, hotels, and facilities with a large movement of people [4].

Significant areas needing antimicrobial protection are materials for sportswear, underwear, and socks. Antimicrobial protection of textiles is also important for technical, home textiles, and sanitary products [4].

Chitosan has been found to be an effective antimicrobial protection for textile materials. The anti-bacterial activity of this polysaccharide is attributed to its amino groups which are crucial for microorganism reduction. In acidic solvents, the amino groups of chitosan become protonated and allow the chitosan, through surface interference, to inhibit the growth of both gram-negative and grampositive bacteria. There are two proposed mechanisms for antibacterial activity of chitosan, both of which emphasise the importance of the amount of active amino groups. In one mechanism, the polycationic nature of chitosan interferes with bacterial metabolism by stacking the cell's surface.

The other mechanism is the binding of chitosan with DNA to inhibit mRNA synthesis [5]. In terms of antimicrobial protection of textile materials [5-14]; however, treatment with chitosan does not always provide the expected results, and on the other hand does not affect some other properties, such as antioxidative or any other action. Namely, the solubility 
of chitosan depends on its purity, level of deacetylation, and the acid used to set the necessary $\mathrm{pH}$ of the medium in which the chitosan is dissolved [5]. For these reasons, it seems an interesting approach to combine chitosan with any of the other natural antimicrobial active components, for example the natural compound called eugenol. Eugenol is an allyl chainsubstituted guaiacol (2-methoxyphenol). It appears as a clear or pale yellow oily liquid. Besides cloves, it can also be extracted from cinnamon and other aromatic spices. Eugenol and its related compounds are proven antioxidants which mean that they inhibit or delay oxidation of other molecules [15-20]. Besides this, eugenol exhibits antiseptic, analgesic, and antiviral properties. Since eugenol is practically insoluble in water, it seems appropriate to synthesize eugenol with chitosan by forming a graft-copolymer of these two compounds [21], which can later be applied to the selected textile substrate in the form of gel. Amino groups of chitosan can therefore be explored as reactive sites for eugenol binding. In this research viscose was used as representative of cellulosic fibers, because it is a biodegradable substrate with good surface activity which, means that in terms of antimicrobial treatment it can be functionalized relatively easily [11, 22]. In addition to the successful synthesis of chitosan/eugenol copolymer and its transfer onto viscose, the selection of treatment temperature is also important. By using the correct temperature, it is possible to establish the conditions for optimally binding copolymer onto the selected substrate.

During this research an FT-IR spectroscopy was used for the synthesis of graft-copolymer chitosan/eugenol evaluation by which the effectiveness of copolymerization is possible to be semiquantitatively analysed. The accessible amino groups can act as dye sites for anionic dyes, therefore method Acid Orange 7 can be explored as an instrument for chitosan (amino groups) amount determination. Furthermore, anti-microbiological testing according to the principle of reducing pathogenic micro-organisms was also included within this research. The obtained results were compared with the results for viscose functionalization with chitosan only, by which good antimicrobial efficiency was confirmed on the basis of previous research work [23, 24].

Journal of Engineered Fibers and Fabrics Volume 9, Issue 3 - 2014

\section{EXPERIMENTAL}

Materials and Methods

\section{Fabric Specification}

Investigations were carried out on $100 \%$ viscose fabric (Lenzing, Austria) with a surface mass of 140 $\mathrm{g} / \mathrm{m}^{2}$, warp density (32 threads / $\mathrm{cm}$ ), filling-density (27 threads / cm), and the weave, (linen).

\section{Pre-Treatment Of Viscose Fabric}

The conventional washing procedure $(1 \mathrm{~g} / \mathrm{L}, \mathrm{T}=$ $40^{\circ} \mathrm{C}, \mathrm{t}=30 \mathrm{~min}$.Sandoclean PC - lineary fatty alcohol of natural origin, high cleaning and rapid wetting power detergent - made by Clariant)) was used in this experiment, plus demineralization. The latter was required in order to remove metal ions and to convert the fibers into their $\mathrm{H}$-forms. Following these treatments, the viscose was rinsed using distilled water until reaching its conductivity $(0.4 /(\mu \mathrm{S} / \mathrm{cm}))$. It was confirmed by the conductivity of the distillate that impurities were effectively removed from the surface of the substrate.

\section{Synthesis of Eugenol/Chitosan}

Grafting of the copolymer chitosan-eugenol was performed according to the following procedure: Chitosan/Aldrich (low molecular weight $\mathrm{Mr}$ 150.000, 90\% de-acetylated) (84 g), and a predetermined amount of eugenol/Sigma-Aldrich (4 x $\left.10^{-3} \mathrm{M}\right)$ based on preliminary tests according literature source [21] was added within a reactor containing $200 \mathrm{~mL}$ of $2 \%$ acetic acid, and then stirred for $4 \mathrm{~h}$ within a nitrogen atmosphere, heated at $40^{\circ} \mathrm{C}$. $6 \times 10^{-3} \mathrm{M}$ ceric ammonium nitrate-CAN/Sigma Aldrich dissolved in $100 \mathrm{~mL}$ of $1 \mathrm{M} \mathrm{HNO}_{3}$, was added within the reactor in order to initiate graft polymerization [21]. The reaction products were precipitated in acetone, filtered, and then used for viscose functionalization.

\section{Chitosan solution preparation}

Dissolution of the chitosan was made with acetic acid, where the $\mathrm{pH}$ was set at 3.6, then continued over 24 hours by stirring at room temperature, using a magnetic stirrer. The complete dissolution of the chitosan was achieved in this way. After the process of impregnation, the samples were dried for $3 \mathrm{~min}$. at $120^{\circ} \mathrm{C}, 150^{\circ} \mathrm{C}$, and $170^{\circ} \mathrm{C}$, respectively. Then, the samples were rinsed at room temperature until reaching the conductivity of the distilled water $(0.4 /(\mu \mathrm{S} / \mathrm{cm}))$. 
Viscose Functionalization with Chitosan and Eugenol/Chitosan

The samples were, firstly immersed for $10 \mathrm{~min}$. in a solution of chitosan or, eugenol/chitosan, and then impregnated with the help of a foulard (W. Mathis AG, Switzerland, $\mathrm{p}=3$ bar, rollers' speed rotation = $2 \mathrm{~m} / \mathrm{min}$ ). 80\% wet pick-up was achieved under these conditions.

\section{ATR FTIR Spectroscopy}

ATR FTIR spectra were recorded on a Perkin Elmer Spectrum GX spectrometer. The ATR accessory contained a diamond crystal. All the spectra (16 scans at $4 \mathrm{~cm}^{-1}$ resolution and rationed to the appropriate background spectrum) were recorded at room temperature.

\section{The Acid Orange 7 Spectrophotometric Method}

This method is based on absorption of the dye C. I. Acid Orange 7 (Figure 1) by the principle of reducing the dye concentration in the dye bath by following the Lambert-Beer Law [22].

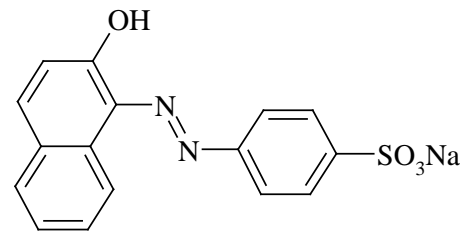

FIGURE 1. Chemical structure of Acid Orange 7 dye.

Sulfonic groups $\left(\mathrm{SO}_{3}^{-}\right)$of the dye form within the acidic medium's ionic bonds in the ratio of 1: 1 with the positively-charged amino groups of chitosan $\left(\mathrm{NH}_{3}{ }^{+}\right)$, so the amount of the dye bound to the fibers corresponds to the amount of accessible amino groups [22]. The initial absorbance at a wavelength of $484 \mathrm{~nm}$, which corresponds to a maximum absorption of the Acid Orange 7 dye, was measured after adding $2 \mathrm{ml}$ of the CI Acid Orange 7 dye (c = $0.005 \mathrm{~mol} / \mathrm{L}$ ) into $250 \mathrm{~mL}$ of water with a $\mathrm{pH}$ of 2.8 . Then $0.25 \mathrm{~g}$ of the fibers was immersed within the dye solution and stirred for three hours at a constant speed, using a magnetic mixer. After a three hour period, the balance between the concentration of the dye in the dye-bath and the concentration of the dye bound on the fibers was established; and the final absorbance was measured using a Cary 50 spectrophotometer, Varian (USA). Based on these measurements of the initial and final absorbance, the concentration of the dye on the fibers was calculated, using Eq.(1):

$A_{i}=\frac{\left(A_{0}-A_{f}\right) \cdot V \cdot 10^{6}}{k \cdot l \cdot m_{f}}$

Journal of Engineered Fibers and Fabrics

Volume 9, Issue 3 - 2014
Where: $A_{i}$ - is the amount of amino groups of chitosan bound to the fibers $/(\mathrm{mmol} / \mathrm{kg}), A_{0}$-intial absorbance, $A_{f}$-final absorbance, $V$ - volume of the dye bath/L, $k$ - correction factor/(L/mol cm), $l-$ length of the optical field/cm, $m_{f}$ - mass of absolutely dry fibers/g.

\section{Antimicrobial Test}

The antimicrobial properties of the treated samples were evaluated at the Institute of Public Health, Maribor, according to ASTM E2149-01, which is a quantitative antimicrobial test method performed under dynamic contact conditions. Escherichia coli, Staphylococcus aureus, and Candida albicans were used as test organisms. An incubated test culture in a nutrient broth was diluted using a sterilized $0.3 \mathrm{mM}$ phosphate buffer $\left(\mathrm{KH}_{2} \mathrm{PO}_{4} ; \mathrm{pH}=6.8\right)$, to give a final concentration of 1.5-3.0 × $10^{5}$ colony-forming units $(\mathrm{CFU}) / \mathrm{mL}$. This solution was used as a working bacterial dilution. Each sample (0.5 to 2 g) was cut into small pieces $(1 \times 1 \mathrm{~cm})$ and transferred into a $250 \mathrm{~mL}$ Erlenmeyer flask containing $50 \mathrm{~mL}$ of the working bacterial dilution. All the flasks were loosely capped, placed in the incubator, and shaken for $1 \mathrm{~h}$ at $37{ }^{\circ} \mathrm{C}$ and $120 \mathrm{rpm}$, using a Wrist-Action incubator shaker. After a series of dilutions using the buffer solutions, $1 \mathrm{~mL}$ of the diluted solution was plated in nutrient agar. The inoculated plates were incubated at $37{ }^{\circ} \mathrm{C}$ for $24 \mathrm{~h}$ and the surviving cells were counted. The average values of the duplicates were converted to $\mathrm{CFU} / \mathrm{mL}$ in the flasks by multiplying by the dilution factor. The antimicrobial activity was expressed as $R=\%$ Eq. (2) the reduction of the organism after contact with the test specimen, compared to the number of bacterial cells surviving after contact with the control [5].

$$
R=\frac{A-B}{A} \cdot 100 \%
$$

Where: $R$ - is the reduction of microbes/\%, $A$ - the number of bacterial colonies after $1 \mathrm{~min}$. (time “ 0 ”), and $B$ - the number of bacterial colonies after 1 hour.

\section{RESULTS \\ ATR FTIR Spectroscopy}

Figure 2 (A) shows the scheme of graft-copolymer chitosan/eugenol, and Figure 2 (B) the FTIR spectrum of chitosan (a), a copolymer of chitozan/eugenol (b), and eugenol (c).The FTIR spectrum of the chitosan showed peaks at 1649 and $1585 \mathrm{~cm}^{-1}$. These two wavenumbers are assigned to the carbonyl stretching vibration (amide I), and the $\mathrm{N}-\mathrm{H}$ bending vibration (amide II) of a primary amino group, respectively. On the other hand Figure 2 (c) 
shows characteristic peaks at 3480,1639 , and 1431 $\mathrm{cm}^{-1}$, which can be attributed to the characteristic peaks of $-\mathrm{OH}$ attached to the benzene, and the $\mathrm{C}=\mathrm{C}$ and $\mathrm{CH}_{2}=$ in the vinyl group, respectively [21]. In the copolymer chitosan-eugenol (Figure 2 b), the formation of a covalent-linkage between the amino group of chitosan, and eugenol was confirmed by the peak shift of amide II in the chitosan to $1526 \mathrm{~cm}^{-1}$ and the disappearance of the free-amino group of chitosan at $1585 \mathrm{~cm}^{-1}$, when compared with chitosan itself. In addition, the characteristic peaks at $1639 \mathrm{~cm}^{-}$ ${ }^{1}(\mathrm{C}=\mathrm{C}), 1431 \mathrm{~cm}^{-1}\left(\mathrm{CH}_{2}=\right)$, and C-H vinyl out-ofplane bending vibrations, observed within the spectrum of the monomer (Figure 2 (c)), had disappeared in the chitosan-eugenol copolymer (Figure 2 (b)). The grafting of the eugenol molecules onto the chitosan was confirmed from the FTIR results.
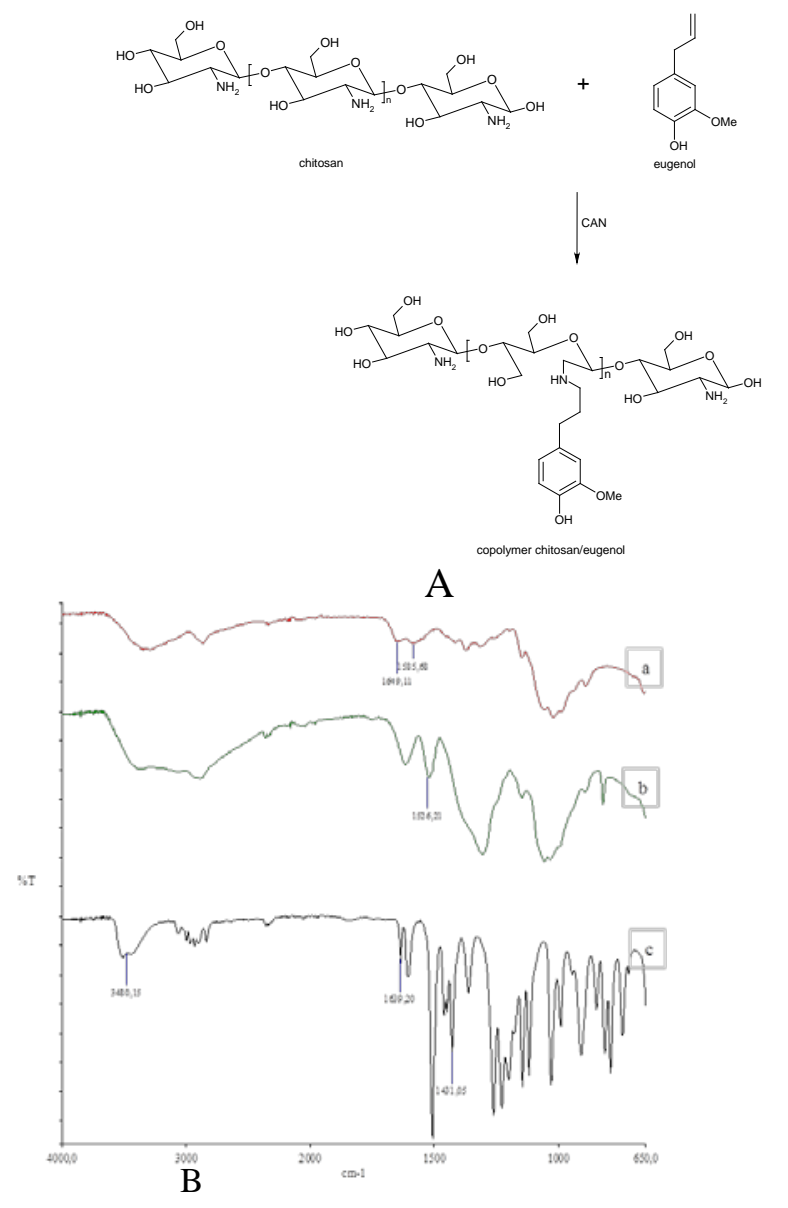

FIGURE 2. Scheme of graft-copolymer chitosan/eugenol (A), and the FTIR spectrum of chitosan (a), a copolymer of chitozan/eugenol (b) and eugenol (c) (B).

\section{Determination of Amino Group Amount}

This indirect method is based on the measure of the anionic dye Acid Orange 7 solution concentration as a result of the dye binding onto the accessible protonated positively- charged amino groups of fibers, which are introduced onto/into the functionalized material (chitosan is fixed onto cellulose by Van der Waals forces, mainly by hydrogen bonds, and lesser by electrostatic interactions [25]). This is seen as an absorbance decrease in comparison with the initial absorbance's value. The accessible amino groups of chitosan are crucial for providing anti-microbial effectiveness; therefore it is desirable to introduce relatively large extent onto/into the material. On the other hand these groups can be explored as binding sites for other substances i.e. eugenol. It reduces the proportion of amino groups; however, additional properties by the combining of two substances are achieved in this way, which is not necessarily reflected as the significantly lower reduction of pathogens. In the sense of providing effective results for viscose functionalization, it is important to select the optimal concentration of chitosan as well as optimizing the drying temperature, in order to assure the successful synthesis of chitosan/eugenol graft copolymer and its bonding with the substrate. Taking into account the preliminary research, where good anti-microbial effectiveness of viscose was proved to be effective by using $1 \%$ solution of chitosan, this concentration was also used for the viscose functionalization of the presented research work. Besides this, the temperatures for drying of 120,150 , and $170^{\circ} \mathrm{C}$ were selected because these temperatures coincide with the theoretical area of the textile material processing of the so-called pad-dry impregnation procedure [23, 24]. In the sense of optimal antimicrobial protection of the textile material needed to reduce a relatively large number of various pathogenic micro-organisms, however, treatment with chitosan sometimes causes problems. Namely, the solubility of chitosan depends on its purity, level of de-acetylation, the acid used to set the $\mathrm{pH}$ medium in which the chitosan should be dissolved, etc. To reduce these problems it is suitable to use low molecular weight product with a degree of de-acetylation near $90 \%$, or higher. Due to eugenol insolubility in an aqueous medium i.e. within an environment which has the optimal condition ( $\mathrm{pH}=3.66)$ for sufficient solubility of the chitosan, the chitosan was combined with eugenol using the synthesis of these two compounds through the formation of the graft-copolymer of these two natural substances powerful against pathogens. After precipitation of the product of reaction in acetone, the formed gel was applied onto the viscose samples, and dried afterwards over a period of $3 \mathrm{~min}$. by using the 
three selected temperatures [26]. The same temperatures and time were also used after treatment of the viscose samples with $1 \%$ chitosan solution only. According to the mechanism of the synthesis (see Figure 2 A), a smaller proportion of the available amino groups, as in the case where for the functionalization only chitosan was used, is expected. This is possible to determine by using the Acid Orange 7 method where the extent of available amine groups' can be relatively easy calculated Eq. (1). The results of the research confirm situation that the proportion of amino group significantly varies depending on the selected drying temperature and is seen to increase with increasing temperature. Therefore, a relatively high drying temperature $\left(170^{\circ} \mathrm{C}\right)$ is recommended for introducing a relatively high proportion of antimicrobially- active amino groups onto the viscose functionalized with copolymer eugenol/chitosan.

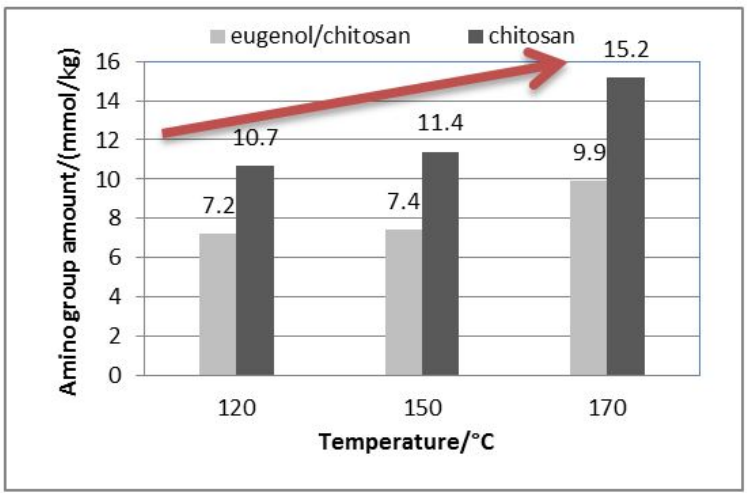

FIGURE 3. Comparison of the available amino group amount of viscose functionalized with copolymer chitosan/eugenol and, viscose functionalized with $1 \%$ solution of chitosan, dried at temperatures of $120^{\circ} \mathrm{C}, 150^{\circ} \mathrm{C}$, and $170^{\circ} \mathrm{C}$.

In the case of non-functionalized cellulose, there were not amino groups present onto/into the viscose which was the reason why this result was excluded from the discussion (Figure 3). In the case of viscose functionalization with $1 \%$ solution of chitosan (Figure 3), it can be seen that after drying at $120^{\circ} \mathrm{C}$ $10.7 \mathrm{mmol} / \mathrm{kg}$, at $150^{\circ} \mathrm{C} 11.4 \mathrm{mmol} / \mathrm{kg}$, and at $170^{\circ} \mathrm{C}$ $15.2 \mathrm{mmol} / \mathrm{kg}$ of available amino groups were introduced into the substrate

The proportion of available amino groups expressed in $\mathrm{mmol} / \mathrm{kg}$, after treatment with copolymer chitosan/eugenol, is smaller than in the case when viscose was functionalized with $1 \%$ chitosan, and is as follows: $120^{\circ} \mathrm{C}-7.2 \mathrm{mmol} / \mathrm{kg} \rightarrow 150^{\circ} \mathrm{C}-7.4$ $\mathrm{mmol} / \mathrm{kg} \rightarrow 170^{\circ} \mathrm{C}-9.9 \mathrm{mmol} / \mathrm{kg}$. The differences in the proportions of available amino groups after viscose being functionalized with $1 \%$ solution of chitosan were $3.5 \mathrm{mmol} / \mathrm{kg}$ after drying at $120^{\circ} \mathrm{C}, 4$ $\mathrm{mmol} / \mathrm{kg}$, after drying at $150^{\circ} \mathrm{C}$ and, $5.3 \mathrm{mmol} / \mathrm{kg}$ after drying at $170^{\circ} \mathrm{C}$. Chitosan solution is a nonNewtonian fluid; therefore, the macromolecules interact with each other and, under certain conditions, tend to form network structures [27]. This phenomenon is also dependent on the processing temperature. Thus, with a higher processing temperature, the macromolecules of chitosan are more likely to interplay with each other in order to form a network. The result is that after cellulose fiber functionalization with $1 \%$ chitosan, a higher proportion of the amino groups was achieved after drying at $170^{\circ} \mathrm{C}$, in comparison with drying at $120^{\circ} \mathrm{C}$ and $150^{\circ} \mathrm{C}$, respectively.

The result of the chitosan/eugenol synthesis was actually seen as a smaller proportion of the available amino groups when compared to the treatment by using $1 \%$ solution of chitosan. This result coincides with the results of FTIR analysis, i.e. indicates the successful synthesis of graft copolymer, where the amino groups actually represent binding sites for eugenol onto the chitosan. Nevertheless, properly antimicrobial activity of the graft copolymer eugenol/chitosan is maintained which can be seen from the further microbiological testing results.

\section{Microbiological Testing}

Microbiological testing of the functionalized viscose on the micro-organisms Escherichia coli (gramnegative non-sporogenes bacteria), Staphylococcus aureus (gram-positive non-sporogenes bacteria), and Candida albicans (fungus) was conducted at the Center for Microbiology at the Institute for Health Protection, Maribor. Before testing, the samples were not sterilized in the sense of achieving asepsis, i.e. a sterile microbial environment due to the fact that materials for sanitary usage are not intended for use within such environments. By using microbiological testing, we wanted to upgrade the results of previous research, and to consider the actual impact regarding the proportion of available amino groups, as well as the effectiveness of the functionalization with the copolymer chitosan/eugenol on the reduction of selected microorganisms. Microbiological testing was performed only after drying at a temperature of $170^{\circ} \mathrm{C}$, whereas the results for determining the proportion of amino groups using the method of Acid Orange VII were the best at this temperature. As is seen from the diagram in Figure 4, the reduction for viscose functionalized with chitosan and the copolymer chitosan/eugenol was effective on the selected micro-organisms and varied within the range of between $96 \%$ and $100 \%$. 
The results show that the functionalized samples inhibited all the selected micro-organisms, where functionalization with $1 \%$ solution of chitosan proved to be slightly more effective in comparison with the functionalization with copolymer chitosan/eugenol. Nevertheless, an excellent antimicrobial activity of the chitosan/eugenol copolymer is recorded after copolymer being applied onto the viscose.

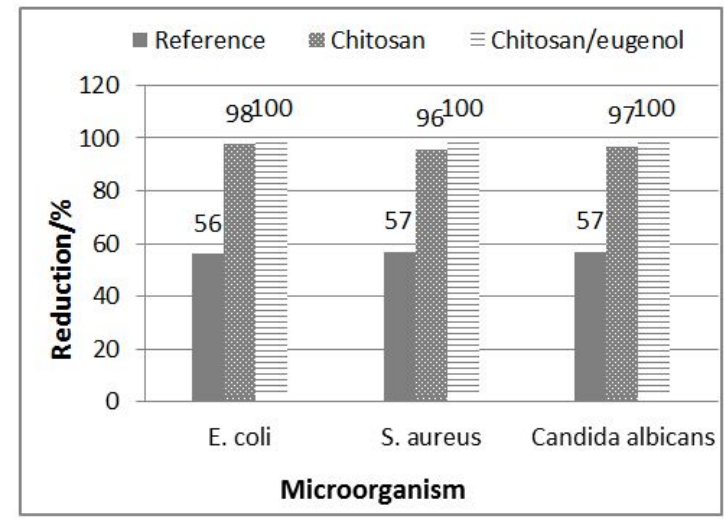

FIGURE 4. Reduction of the micro-organisms of nonfunctionalized viscose, viscose functionalized with $1 \%$ solution of chitosan and viscose, and functionalized with copolymer chitosan/eugenol, after drying at $170^{\circ} \mathrm{C}$.

Microbiological testing also showed a reduction in the selected pathogenic micro-organisms in the case of the reference sample (non-functionalized viscose) due to the substrate properties.

The fabric (also viscose) can roughly be considered as a material with a net structure; therefore, the micro-organisms were adsorbed relatively easily onto such a substrate. For this reason the used method could not be considered as a completely reliable test. The used test method was based on the taking of the sample from the solution, which meat that the result was influenced by the method of sampling, i.e. if the sample was taken at the surface of the material, or within its immediate environment.

However, in the case of the reference samples, there was insufficient reduction of the micro-organisms used in this study because, on the basis of the test methods prescribed by the standard ASTM E 214901 on the successful reduction of $(\mathrm{R} / \%)$ on microorganisms, it is only possible to conclude this in the case where the reduction is higher than 75\% [5].

On the basis of the pathogenic micro-organisms' inhibition it can be concluded that a very good total reduction of pathogenic micro-organisms was achieved with both procedures of functionalization.

\section{CONCLUSION}

The conclusion of this study is that both materials (functionalized by $1 \%$ chitosan solution and copolymer eugenol/chitosan) show excellent antimicrobial capacity against Gram positive and negative bacteria as well as fungi. This means that, despite the reduction of amino groups as a result of the formation of chitosan/eugenol graft copolymer excellent antimicrobial properties are maintained. Considering the additional properties of eugenol as its anti-oxidative, antimycotic action, the combination of eugenol/chitosan is considered to be a better choice in terms of a more diverse protection of textile materials against pathogenic microorganisms. In addition the following were concluded:

- The successful synthesis of graft-copolymer containing chitosan/eugenol was confirmed using ATR FTIR spectroscopy.

- The proportion of amino groups significantly varied depending on the selected temperature and increased with an increasing drying temperature. Temperature of drying of $170^{\circ} \mathrm{C}$ is recommended for introducing relatively high proportion of antimicrobial acting amino groups onto the viscose functionalized with copolymer eugenol/chitosan.

- The proportion of available amino groups, expressed in $\mathrm{mmol} / \mathrm{kg}$ was, after treatment with copolymer chitosan/eugenol, smaller than in the case where viscose was functionalized by using $1 \%$ solution of chitosan. These results coincide with the FTIR results and due to amino group amount decrease indicate the successful synthesis of graft copolymer.

- Considering the additional properties of eugenol as its anti-oxidative, antimycotic action, the combination of eugenol/chitosan is considered to be a better choice in terms of a more diverse protection of textile materials against pathogenic microorganisms.

- Finally, it is necessary to consider the fact that micro-organisms will eventually become resistant to antimicrobial active compounds, and it is therefore necessary to find new possibilities for effective protection of textile materials against microorganisms through the use of a copolymer of eugenol/chitosan, which had been proven to be a very interesting and successful combination of these two natural compounds. 


\section{REFERENCES}

[1] Briozzo, J. et al., Antimicrobial activity of clove oil dispersed in a concentrated sugar solution, Journal of Applied Bacteriology, 66, 69-75 (1989).

[2] Chuenchit B. and Flegel T.W., In vitro antifungal activity of eugenol and vanillin against Candida albicans and Cryptoccocusneo formans, Canadian Journal of Microbiology, 28, 1235-1241 (1981).

[3] Didry N. et al., Activity of thymol, carvacrol, cinnamal dehyde and eugenol on oral bacteria, Pharmaceutica Acta Helvetiae, 69, 25-28 (1994).

[4] Šauperl O. and Fras-Zemljič L., Textiles for protection and sport, Faculty of Mechanical Engineering, Maribor, (2011).

[5] Fras-Zemljič L. et al., Viscose material functionalized by chitosan as a potential treatment in gynaecology, Textile Research journal, 81(11), 1183-1190 (2011).

[6] Rajendran S. et al., Developments in Medical Textiles: a critical appreciation of recent developments, Textile Progress, 32(4), (2002).

[7] Majeti N. V. and Ravi Kumar M. N. V., A review of chitin and chitosan applications, Reactive \& Functional Polymers, 46(1), 1-27 (2000).

[8] Fras-Zemljič L. et al., Chitosan coatings onto polyethylene terephthalate for the development of potential active packaging material, Appl. surf. sci., 265, 697-703 (2013).

[9] Fras-Zemljič L. e tal., Characterization of regenerated cellulose fibers antimicrobial functionalized by chitosan, Textile research. journal., 83(2), 185-196 (2013).

[10] Fras-Zemljič L. and Šauperl O., Chitosan and its derivatives as an adsorbate for cellulose fibres' anti-microbial functionalizations, Industria. textila, 63(6), 296-301 (2012).

[11] Strnad S. et al., Cellulose fibres functionalised by chitosan: characterization and application, V: ELNASHAR, Magdy M. (ur.). Biopolymers, Rijeka: Sciyo, cop., 181-200 (2010).

[12] Strnad S. et al., Chitosan - a universally applicable biopolymer, Tekstilec, 50(10/12), 243-261 (2007).

[13] Strnad S. et al., Influence of chemical modification on sorption and mechanical properties of cotton fibers treated with chitosan, Textile research. Journal, 78(5), 390-398 (2008).
[14] Vodišek N., Functionalization of Fibres for Bioactive Properties, Research work, Maribor, (2010).

[15] Hao Y. Y. et al., Efficacy of plant extracts in inhibiting Aeromonashy drophila and Lysteriamonocytogenes in refrigerated, cookedpoultry, Food Microbiology, 15, 367378 (1998).

[16] Mahmoud A. L. E, Antifungal action and antiaflatoxigenic properties of some essential oil constituents, Letters in Applied Microbiology, 19, 110-113 (1994).

[17] Quattara B. et al., Antibacterial activity of selected fatty acids and essential oils against sixmeat spoilage organisms, International Journal of Food Microbiology, 37, 155-162 (1997).

[18] Shapiro S. et al., The antimicrobial activity of essential oils and essential oil components towards oral bacteria, Oral Microbiology and Immunology, 9, 202-208 (1994).

[19] Subrayam C. and Jayashree T., Antiaflat toxigenic activity of eugenol is due to inhibition of lipid peroxidation, Letters in Applied Microbiology, 28, 179-183 (1999).

[20] Zemek J. et al., Antimicrobial properties of aromatic compounds of plantorigin, Folia Microbiolgica, 32, 421-425 (1987).

[21] Byung-Ok J. et al., Preparation and characterization of Eugenol-Grafted Chitosan Hydrogels and Their Antioxidant Activities, Journal of Applied Polymer Science, 99, 3500-3506 (2006).

[22] Fras-Zemjič L. et al., Amino groups determination on chitosan treated cotton fibres by spectrometrical titration techniques. V: EPNOE Scientific Meeting, Iasi, Romania, (2007).

[23] Šauperl O. and Stana-Kleinschek K., Differences between cotton and viscose fibers crosslinked with BTCA, Textile Research Journal, 80(4), 383-392 (2010).

[24] Schindler, W. D., Hauser P. J., Chemical finishing of textiles. Cambridge England, Woodhead Publishing Limited, (2004).

[25] Čakara, D. et al., Protonation behavior of cotton fabric with irreversibly adsorbed chitosan: a potentiometric titration study, Carbohydr. Polymers, 78 (1), 36-40 (2009).

[26] Khokhlova, M. A. et a., Chitosan macromolecules on a substrate: deposition from solutions in sc $\mathrm{CO}_{2}$ and reorganisation in vapours, Macromolecular symposia, 269, 531-540 (2010). 
[27] Šauperl, O., Cotton cellulose crosslinking monitored by different physical-chemical methods, dissertation, Maribor, (2004).

\author{
AUTHORS' ADDRESSES \\ Olivera Sauperl \\ Jasna Tompa \\ Julija Volmajer-Valh \\ Faculty of Mechanical Engineering \\ Smetanova Ulica 017 \\ Maribor, Slovenia SI 2000 \\ SLOVENIA
}

